\title{
复合莫来石纤维前驱体结晶行为研究
}

\author{
马西飞，林根连，康 庄，黄 晓 \\ (中国科学院 上海硅酸盐研究所, 上海 200050)
}

摘 要: 无玻璃相微晶结构是莫来石纤维获得良好高温力学性能的关键, 采用单/双相莫来石复合前驱体可在不添 加玻璃相物质前提下，调控莫来石纤维结晶结构。在复合前驱体中，单相莫来石组分在 $980^{\circ} \mathrm{C}$ 原位生成的莫来石晶 粒可作为晶核，诱导双相莫来石组分在较低的温度下形成莫来石晶相，使前驱体具有较好的结晶特性及致密化特 性，并获得微晶、均匀、致密的结构。采用单、双相莫来石前驱体混合的方法制备了一系列可纺复合莫来石前驱体， 通过 XRD、DSC 及 SEM 对其高温莫来石结晶行为进行了研究, 并据此确定了最佳的复合前驱体组成。研究发现, 含 $70 \mathrm{wt} \%$ 单相组分的复合前驱体具有最好的结晶及致密化特性，与双相莫来石前驱体相比，经 $1400^{\circ} \mathrm{C}$ 热处理 $20 \mathrm{~h}$ 后， 其晶核密度从 $8 \times 10^{12} / \mathrm{cm}^{3}$ 提高到 $1 \times 10^{15} / \mathrm{cm}^{3}$, 平均粒径从 $360 \mathrm{~nm}$ 降至 $120 \mathrm{~nm}$ 。

关 键 词: 莫来石纤维; 复合莫来石前驱体; 结晶行为

中图分类号: TQ050 文献标识码: A

\section{Crystallization Behavior of Hybrid Mullite Fiber Precursors}

\author{
MA Xi-Fei, LIN Gen-Lian, KANG Zhuang, HUANG Xiao
}

(Shanghai Institute of Ceramics, Chinese Academy of Sciences, Shanghai 200050, China)

\begin{abstract}
Glass free fine-grained microstructure is ideal for preparing mullite fibers with good high temperature mechanical properties. Single phase/diphasic mullite hybrid precursor was adopted to adjust crystalline microstructure of mullite fibers without adding any glass formers. The single phase precursor crystallized in situ to form mullite at $980^{\circ} \mathrm{C}$ acting as seed crystals for diphasic components in hybrid precursors to induce the formation of mullite crystals at low temperatures. A series of spinnable hybrid mullite precursors were prepared by mixing single phase and diphasic mullite precursors, and their high temperature crystallization behaviors were investigated by XRD, DSC and SEM, with which optimum formulation of hybrid precursor was determined. The results reveal that hybrid precursor containing $70 \mathrm{wt} \%$ single phase component has ideal crystalline properties and densification characteristics, with fine grain, uniform, and dense structure. Compared with the pure diphasic mullite precursor, the introduction of $70 \mathrm{wt} \%$ single phase precursor resulted in an increase in apparent nucleation from $8 \times 10^{12} / \mathrm{cm}^{3}$ to $1 \times 10^{15} / \mathrm{cm}^{3}$, and a reduction in grain size from $360 \mathrm{~nm}$ to $120 \mathrm{~nm}$ after heat-treatment at $1400^{\circ} \mathrm{C}$ for $20 \mathrm{~h}$.
\end{abstract}

Key words: mullite fiber; hybrid mullite precursor; crystallization behavior

莫来石纤维的化学组成与莫来石 $\left(3 \mathrm{Al}_{2} \mathrm{O}_{3} \cdot 2 \mathrm{SiO}_{2}\right)$ 的接近, 与其他氧化铝基纤维相比, 它具有高温强 度保持率好、抗蠕变性能及抗热震稳定性高的特点,
应用十分广泛，是氧化铝基陶瓷纤维中产量最大的 品种 ${ }^{[1-4]}$ 。以莫来石纤维制备的构件及复合材料在 航空和航天领域获得了广泛应用。在工业领域中,

收稿日期：2016-11-08; 收到修改稿日期：2016-12-21

基金项目：国家自然科学基金(51302289); 上海市自然科学基金(13ZR1445800)

National Natural Science Foundation of China(51302289); National Science Foundation of Shanghai(13ZR1445800)

作者简介：马西飞(1989-)，男，硕士，助理工程师. E-mail: maxifei@126.com

通讯作者：康 庄, 副研究员. E-mail: kangz@mail.sic.ac.cn 
莫来石纤维可用于制作高温炉窑的炉衬、炉窝、高 温密封垫圈以及热电偶保护套管等制件 ${ }^{[5-8]}$ 。

控制莫来石纤维结晶结构是获得高性能纤维的 关键 ${ }^{[9-12]}$ 。目前制备莫来石纤维前驱体主要是双相 莫来石前驱体(diphasic mullite gel $)^{[9,13]}$ 。在这类前驱 体中, 铝、硅组分在纳米级尺度上均匀混合, 体系莫 来石化温度高于致密化温度。前驱体在热处理过程 中, 体系首先在无定形氧化硅导致的过渡粘性烧结 机制下完成致密化, 而后体系内的 $\gamma$-氧化铝与氧化 硅在 $1200^{\circ} \mathrm{C}$ 以上反应生成莫来石结晶。双相前驱体 虽在致密化方面具有优势, 但其较高的莫来石化温 度会使纤维内莫来石晶粒在高温下过度生长, 导致 纤维强度降低, 脆性增加 ${ }^{[14]}$ 。目前常用的抑制莫来 石纤维晶粒生长的方法是在纤维内添加氧化硼等玻 璃相物质, 高温下氧化嗍能够大幅降低氧化硅粘度, 提高物质扩散速度, 加速莫来石结晶成核, 从而降 低莫来石结晶成核的势垒。但氧化嗍的引入也会对 纤维的高温性能带来不利的影响: 一方面, 氧化嗍 在 $1200^{\circ} \mathrm{C}$ 以上挥发会对纤维结构造成严重破坏; 另 一方面, 氧化嗍的加入在纤维内引入玻璃相, 使纤 维在高温下发生蠕变, 劣化纤维在高温下的力学性 能 ${ }^{[15-17]}$ 。如何使莫来石纤维获得微晶无玻璃相的理 想结构, 是提高莫来石纤维高温力学性能需要解决 的关键问题。

在单相莫来石前驱体(single phase mullite gel) 中, 硅、铝组分在分子水平上均匀混合, 并在 $980^{\circ} \mathrm{C}$ 直接生成莫来石结晶, 其莫来石化温度低于致密化 温度, 致密化特性很差, 无法用于纤维制备。Huling 和 Messing 等 ${ }^{[18-19]}$ 通过在双相莫来石前驱体中引入 单相莫来石前驱体的方法, 对莫来石陶瓷进行了结 晶结构的调控。利用单相莫来石前驱体原位生成莫 来石晶粒作为晶核, 降低双相莫来石前驱体的成核 势垒, 使其在较低的温度下形成莫来石相, 获得均 匀细晶的显微结构。他们研究发现, 在双相莫来石 胶体中加入 $30 \mathrm{wt} \%$ 的单相胶体, 可使体系 $1550^{\circ} \mathrm{C}$ 处 理后的晶粒尺寸由 $1.4 \mu \mathrm{m}$ 下降至 $0.4 \mu \mathrm{m}$ 。上述工作 为莫来石纤维结晶结构调控提供了思路, 但其采用 的是具有触变性的勃姆石胶体制备莫来石前驱体, 因此无法直接应用于纤维的制备。目前, 尚没有研 究将这一方法应用于莫来石纤维的制备。可纺性莫 来石前驱体的化学组成、胶体结构有其自身特点, 结晶行为也与上述体系有所差别, 因此需要进行更 细致的研究来获得最佳的复配比例。本工作采用可 纺双相莫来石前驱体与单相莫来石前驱体复合, 制 备了一系列可纺性良好的复合莫来石前驱体, 研究
了不同单、双相前驱体组成对复合前驱体莫来石结 晶行为及晶粒结构的影响, 为无玻璃相微晶莫来石 纤维的制备提供指导。

\section{1 实验方法}

\section{1 双相莫来石前驱体的制备}

将硝酸铝(AN)溶解于去离子水中, 在快速搅拌下 加入异丙醇铝(AIP), 室温下反应 $20 \mathrm{~h}$ 直至异丙醇铝完 全溶解, 将所得溶胶转移至夹套水浴反应釜中升温 至 $90^{\circ} \mathrm{C}$, 反应 $12 \mathrm{~h}$ 得到铝溶胶。控制上述体系中各 种物质的摩尔比为 $n(\mathrm{AIP}): n(\mathrm{AN}): n\left(\mathrm{H}_{2} \mathrm{O}\right)=9: 3$ : 180 。在快速摚拌条件下, 将粒子型水性硅溶胶(浙 江宇达化工有限公司 NS-30 型)逐渐加入到上述铝 溶胶中, 控制 $m\left(\mathrm{Al}_{2} \mathrm{O}_{3}\right): m\left(\mathrm{SiO}_{2}\right)=72: 28$, 得到双 相莫来石溶胶。

\section{2 单相莫来石前驱体的制备}

将硝酸铝(AN)溶解于去离子水中, 然后在快速 搅拌下依次加入异丙醇铝(AIP) 和正硅酸乙酯 (TEOS), 室温下反应 $20 \mathrm{~h}$ 至异丙醇铝完全溶解, 将 溶液转移至夹层水浴反应釜中升至 $90^{\circ} \mathrm{C}$, 反应 $12 \mathrm{~h}$ 得到单相莫来石溶胶。控制上述溶液中各物质的摩 尔比为 $n(\mathrm{AIP}): n(\mathrm{AN}): n(\mathrm{TEOS}): n\left(\mathrm{H}_{2} \mathrm{O}\right)=9: 3:$ $4: 180$ 。

\section{3 复合前驱体的制备}

双相莫来石溶胶与单相莫来石溶胶按照表 1 所 述比例混合, 得到复合莫来石前驱体溶胶。

\section{4 莫来石前驱体的表征}

使用 DSC(STA 449F3, Netzsch)以 $10 \mathrm{~K} / \mathrm{min}$ 的升 温速度跟踪莫来石前驱体溶胶在空气中 $40 \sim 1500^{\circ} \mathrm{C}$ 的热裂解过程; 不同组分的复合溶胶在 $1100^{\circ} \mathrm{C}$ 、 $1200^{\circ} \mathrm{C} 、 1300^{\circ} \mathrm{C}$ 热处理 $1 \mathrm{~h}$ 后，使用 $\mathrm{X}$ 射线衍射仪 (D/max-2250, Rikagu) 分析其结晶结构; 使用扫描电 镜(SU8820, Hitachi)观察分析热处理后样品的形貌 及晶粒大小。

表 1 不同比例复合溶胶的组成

Table 1 Compositions of hybrid mullite precursors

\begin{tabular}{ccc}
\hline Sample & Single phase gel $/$ wt $\%$ & Diphasic gel $/$ wt $\%$ \\
\hline S & 100 & 0 \\
s9d1 & 90 & 10 \\
s7d3 & 70 & 30 \\
s5d5 & 50 & 50 \\
s3d7 & 30 & 70 \\
s1d9 & 10 & 90 \\
D & 0 & 100
\end{tabular}




\section{2 结果与讨论}

\section{1 不同组分复合前驱体结晶温度变化}

图 1 是莫来石前驱体溶胶的 DSC 曲线, 对比各 组成溶胶 DSC 曲线可知: 单相莫来石前驱体溶胶在 $987.19^{\circ} \mathrm{C}$ 有唯一的尖锐的放热峰, 此峰对应富铝四 方相莫来石结晶峰。文献[18]研究表明: $\mathrm{Si}-\mathrm{O}-\mathrm{Al}$ 键 在单相前驱体化学键中的比例越高, DSC 升温速度 越慢, 前述转变温度越接近 $980^{\circ} \mathrm{C}$ 。本研究制备的单 相前驱体溶胶莫来石转变温度仅比理论温度高 $7^{\circ} \mathrm{C}$, 说明该单相莫来石前驱体溶胶中硅、铝组分达到分 子层级混合。双相莫来石前驱体的莫来石温度在 $1318.18^{\circ} \mathrm{C}$, 此温度远高于单相莫来石前驱体莫来石 化温度, 这主要是由于双相莫来石前驱体溶胶中 硅、铝在纳米尺度混合, 大尺寸级别的混合导致物 质传输距离上增加, 同时物质间相互隔绝作用加剧, 所以需要更高的温度才能产生莫来石相。复合前驱 体 s1d9、s3d7、s5d5、s7d 3 的莫来石化温度比双相 莫来石前驱体分别低 $19^{\circ} \mathrm{C} 、 26^{\circ} \mathrm{C} 、 55^{\circ} \mathrm{C} 、 73^{\circ} \mathrm{C}$, 表 明复合莫来石前驱体莫来石化温度随体系中单相前 驱体的增加而降低。单相莫来石前驱体的比例提高 到 $90 \%$ 时, 复合前驱体的 DSC 曲线更接近于单相前 驱体。单相莫来石前驱体所占比例是预测复合莫来 石前驱体莫来石化温度的一个重要指标。物质的生 成和转变不仅仅依赖热力学因素, 动力学因素也是 物质生成的必要条件。如果生长动力足够, 且亚稳 定相优先稳定相成核, 那么就有可能先生成亚稳定 相。在复合莫来石前驱体体系内, 双相莫来石前驱 体在 $500^{\circ} \mathrm{C}$ 左右生成 $\gamma-\mathrm{Al}_{2} \mathrm{O}_{3}$ 。由于 $\gamma-\mathrm{Al}_{2} \mathrm{O}_{3}$ 和硅铝 尖晶石具有相似晶胞格子, 硅铝尖晶石在 $\gamma-\mathrm{Al}_{2} \mathrm{O}_{3}$ 存在的条件下优先生成, 当硅铝尖晶石形成后, 大 量单相莫来石前驱体被消耗在尖晶石晶粒的生长, 莫来石相的生成受到很大程度的抑制 ${ }^{[6,19]}$ 。复合莫

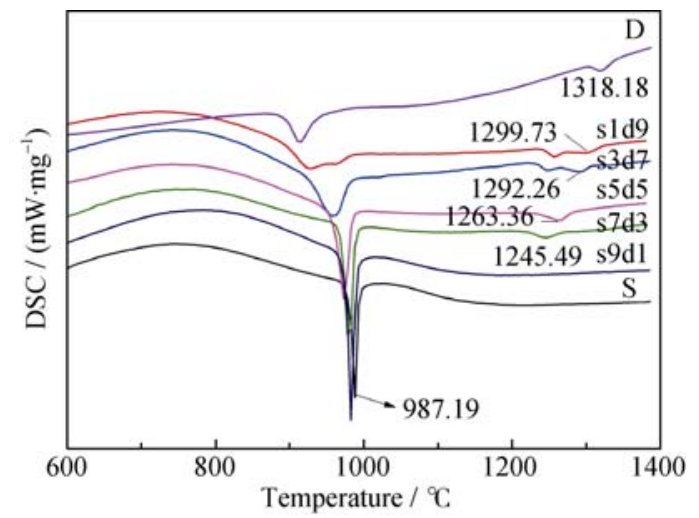

图 1 不同配比莫来石前驱体的 DSC 曲线

Fig. 1 DSC curves for hybrid mullite precursors
来石前驱体中含有一定量的双相莫来石组分，会造 成其莫来石化温度的显著升高。

\section{2 不同组成复合前驱体结晶相变路径}

图 2 分别是莫来石前驱体经 $1100^{\circ} \mathrm{C} 、 1200^{\circ} \mathrm{C}$ 、 $1300^{\circ} \mathrm{C}$ 热处理 $1 \mathrm{~h}$ 产物的 XRD 图谱。图 2(a)显示经 $1100^{\circ} \mathrm{C}$ 热处理 $1 \mathrm{~h}$ 后, 单相莫来石前驱体完全转变为 莫来石相; 复合莫来石前驱体 $\mathrm{s} 9 \mathrm{~d} 1 、 \mathrm{~s} 7 \mathrm{~d} 3$ 不仅存在莫 来石相，而且存在硅铝尖晶石相; 复合莫来石前
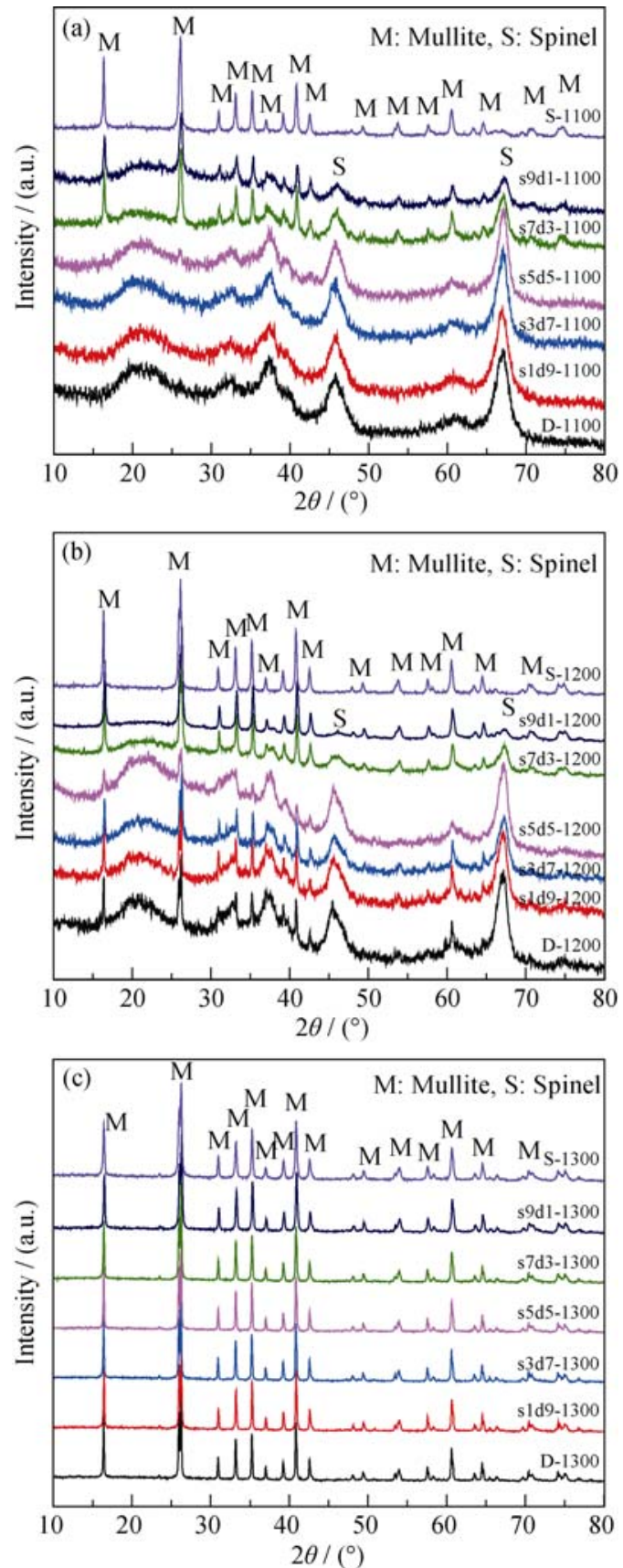

图 2 不同配比莫来石前驱体经 $1100^{\circ} \mathrm{C}$ (a)、 $1200^{\circ} \mathrm{C}(\mathrm{b})$ 和 $1300^{\circ} \mathrm{C}(\mathrm{c})$ 热处理 $1 \mathrm{~h}$ 产物的 XRD 图谱

Fig. 2 XRD patterns of the products from mullite precursors calcined at $1100^{\circ} \mathrm{C}(\mathrm{a}), 1200^{\circ} \mathrm{C}(\mathrm{b})$ and $1300^{\circ} \mathrm{C}(\mathrm{c})$ for $1 \mathrm{~h}$ 
驱体 s1d9、s3d7、s5d5 和双相莫来石前驱体仅存在 尖晶石相。此结果进一步印证了 DSC 结果。随着单 相莫来石前驱体比例的增加, 复合莫来石前驱体莫 来石化温度越低; 当单相莫来石前驱体在复合莫来 石前驱体中所占比例低于 $50 \%$ 时, $1100^{\circ} \mathrm{C}$ 热处理 $1 \mathrm{~h}$ 无法形成莫来石相。复合莫来石前驱体中双相莫来 石前驱体形成的尖晶石晶粒生长消耗了大量的单相 莫来石前驱体。单相莫来石前驱体欲达到自成核诱 导莫来石相的生成, 不仅要提供足够尖晶石的生长 量, 而且需要保留有部分能够进行自成核。当单相 莫来石前驱体所占比例达到一定程度才能降低复合 莫来石前驱体的莫来石化温度。

图 2(b)显示经 $1200^{\circ} \mathrm{C}$ 热处理 $1 \mathrm{~h}$ 后, 单相莫来 石前驱体、复合莫来石前驱体及双相莫来石前驱体 均有莫来石相生成, 但仅单相莫来石前驱体得到纯 相莫来石; s9d1、s7d3 前驱体的莫来石相峰高有较 大程度的增加, 表明体系内莫来石晶相比例增加。 图 2(c)显示经 $1300^{\circ} \mathrm{C}$ 热处理 $1 \mathrm{~h}$ 后, 所有前驱体均 完全莫来石化。综合 XRD 分析, s7d 3 前驱体体系在 $1100 \sim 1200^{\circ} \mathrm{C}$ 温度区间内热处理不仅有莫来石相生
成，同时在体系内还保留有相当比例的硅铝尖晶石 相和无定型氧化硅，因此其具有较好的致密化及结 晶特性。

\section{3 不同莫来石前驱体显微结构比较}

图 3 为双相莫来石前驱体、 $\mathrm{s} 7 \mathrm{~d} 3$ 复合前驱体经 $1400^{\circ} \mathrm{C}$ 处理 $20 \mathrm{~h}$ 后产物的扫描电镜照片及晶粒尺寸统 计图。对比图 3(a)、(b)可知, 在 $1400^{\circ} \mathrm{C}$ 热处理 $20 \mathrm{~h}$ 后, 双相莫来石前驱体中莫来石晶粒平均粒径为 $360 \mathrm{~nm}$, 且粒径分布较宽; $s 7 d 3$ 复合莫来石晶粒平均粒径在 $\sim 120 \mathrm{~nm}$, 且粒径均一, 分布较窄。通过计算, 双相莫 来石前驱体晶核密度为 $8 \times 10^{12} / \mathrm{cm}^{3}$, 而复合莫来石前 驱体 $\mathrm{s} 7 \mathrm{~d} 3$ 晶核密度为 $1 \times 10^{15} / \mathrm{cm}^{3}$ 。由 SEM 分析可 知，单相组分的加入有效地增加了体系内晶核的密 度, 使前驱体获得微晶均一的微观结构。相关纤维 制备及高温性能的研究正在进行中, 初步结果显示, 采用 $\mathrm{s} 7 \mathrm{~d} 3$ 前驱体制备的莫来石纤维，经 $1300^{\circ} \mathrm{C}$ 热处 理 $1 \mathrm{~h}$ 后纤维强度保持率比双相前驱体莫来石纤维 提高约 $10 \%$ 。
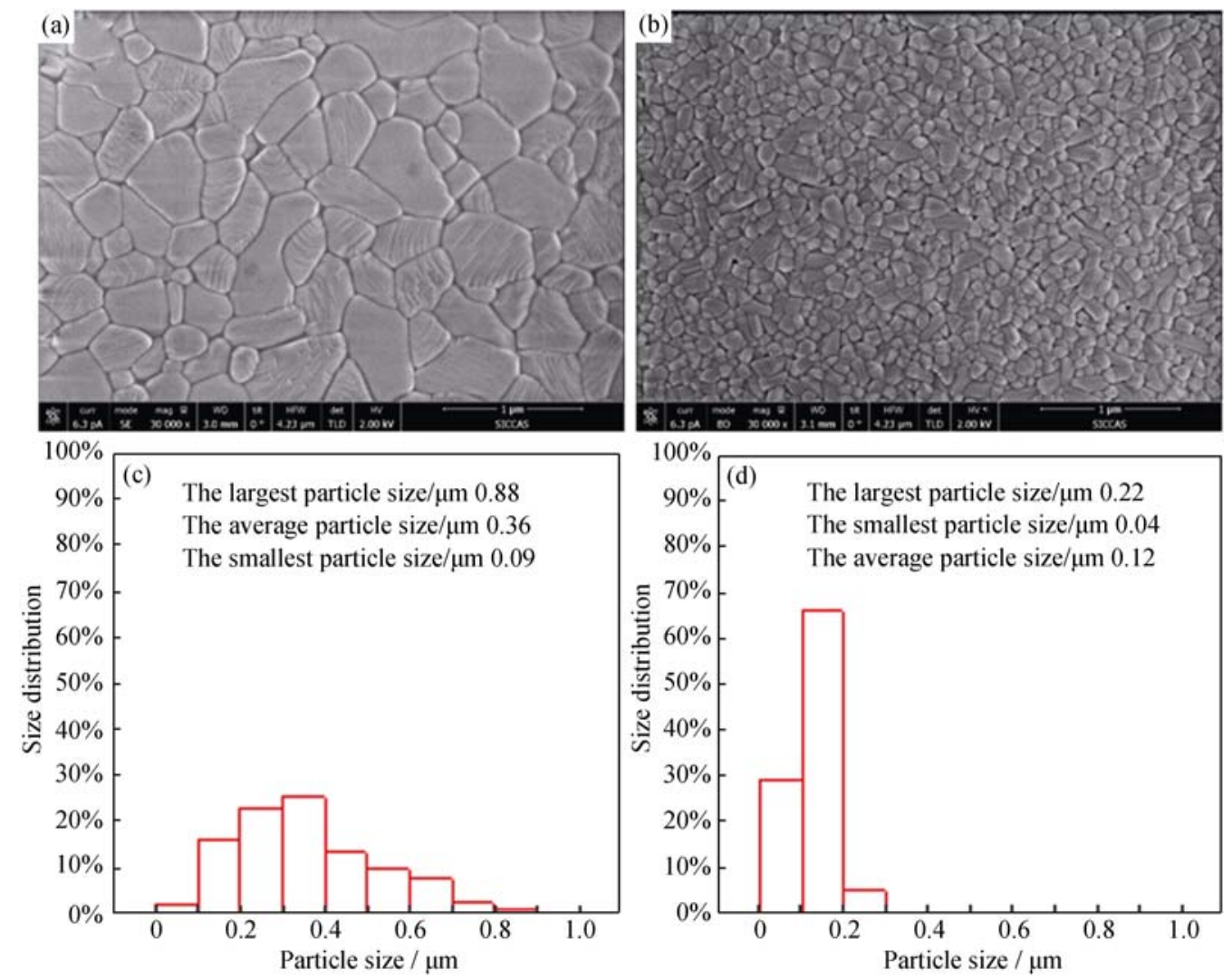

图 3 莫来石前驱体 $1400^{\circ} \mathrm{C} 20 \mathrm{~h}$ 热处理后扫描电镜图片及晶粒尺寸分布图

Fig. 3 High magnification SEM images and crystal size distribution statistical graph of mullite precursors calcined at $1400^{\circ} \mathrm{C}$ for $20 \mathrm{~h}$ (a, c) Diphasic mullite precursor; (b, d) s7d3 hybrid mullite precursor 


\section{3 结论}

1)单/双相复合莫来石前驱体中，单相莫来石 组分低温下原位生成的莫来石晶粒作为晶核可以 显著降低双相组分的成核势垒，增加体系内晶核 的密度, 从而使体系获得微晶且晶粒尺寸均匀的 理想微观结构。

2)复合莫来石前驱体中, 双相莫来石组分在较 低温度下生成 $\gamma-\mathrm{Al}_{2} \mathrm{O}_{3}$, 随后产生硅铝尖晶石, 硅铝 尖晶石晶粒生长消耗了大量的单相莫来石组分。因 此复合莫来石前驱体中需含有 $50 \mathrm{wt} \%$ 以上的单相组 分才能显著降低体系的莫来石化温度。

3)与双相莫来石前驱体相比, 含 $70 \mathrm{wt} \%$ 单相组 分的复合莫来石前驱体经 $1400^{\circ} \mathrm{C}$ 热处理 $20 \mathrm{~h}$ 后, 晶 核密度从 $8 \times 10^{12} / \mathrm{cm}^{3}$ 提高到 $1 \times 10^{15} / \mathrm{cm}^{3}$, 晶粒平均 粒径从 $360 \mathrm{~nm}$ 降至 $120 \mathrm{~nm}$ 。初步结果显示, 采用 该比例复合前驱体制备的莫来石纤维，经 $1300^{\circ} \mathrm{C}$ 热 处理 $1 \mathrm{~h}$ 后纤维强度保持率比双相前驱体莫来石纤 维提高约 $10 \%$ 。

\section{参考文献:}

[1] OHNABEA H, MASAKIA S, ONOZUKAA M, et al. Potential application of ceramic matrix composites to aero-engine components. Compos Part A, 1999, 30(4): 489-496.

[2] KAYA C, KAYA F, BUTLER E G. Development and characterization of high-density oxide fiber-reinforced oxide ceramicmatrix composites with improved mechanical properties. Journal of the European Ceramic Society, 2009, 29(9): 1631-1639.

[3] CIVIDANE S L, CAMPOST M B, RODRIGUES L A. Review of mullite synthesis routes by Sol-Gel method. Journal of Sol-Gel Science and Technology. 2010, 55(1): 111-125.

[4] DOKKO P C, PASK J A, MAZDIYASNI K S. High-temperature mechanical properties of mullite under compression. Journal of the American Ceramic Society. 1977, 60(3/4): 150-155.

[5] 乔 健, 刘和义, 崔宏亮. 莫来石纤维制备方法综述. 硅酸盐通 报, 2014, 33(12): 3230-3234.

[6] JIN XI-HAI, GAO LIAN, GUO JING-KUN. Preparation of mullite powders by Sol-Gel methods. Journal of Inorganic Materials,
2001, 16(3): 555-558.

[7] 王淑峰, 刘立强, 姚树玉。连续莫来石陶瓷纤维的制备研究。硅 酸盐通报.2006, 25(3): 15-19.

[8] CHEN X, GU L X. Structural evolution of Sol-Gel derived mullite fibers with different solid contents during sintering. Journal of Materials Pro cessing Technology.2009, 209(8): 3991-3998.

[9] OKADA K, YASOHAMAS, HAYASHI S. Sol-Gel synthesis of mullite long fibres from water solvent systems. Journal of the European Ceramic Society, 1998, 18(13): 1879-1884.

[10] ZHANG Y, DING P, GAO J. Mullite fibres prepared by Sol-Gel method using polyvinyl butyral. Journal of the European Ceramic Society, 2009, 29(6): 1101-1107.

[11] ALMEDIA R S M, TUSHTEV K, CLAUß B. Tensile and creep performance of a novel mullite fiber at high temperatures. Composites: Part A, 2015, 76: 37-43.

[12] ALMEDIA R S M, BERGMULLER E L, EGGERT B G F. Thermal exposure effects on the strength and microstructure of a novel mullite fiber. Journal of the American Ceramic Society, 2016, 99(5): 1709-1716.

[13] LI CHENG-SHUN, ZHANG YU-JUN, ZHANG JING-DE. Polycrystalline mullite fibers prepared by Sol-Gel method. Journal of Inorganic Materials, 2009, 24(4): 848-852.

[14] SCHNEIDER H, KOMMAMENI S. Mullite Fibers, in Mullite. Wiley-VCH Verlag GmbH\&KgaA, 2006: 377-396.

[15] SCHMUCKER M, FLUCHT F, SCHNEIDER H. High temperature behaviour of polycrystalline aluminosilicate fibres with mullite bulk composition. I. Microstructure and strength properties. Journal of the European Ceramic Society, 1996, 16(2): 281-285.

[16] HILDMANN B O, SCHNEIDER H, SCHMUCKER M. High temperature behaviour of polycrystalline aluminosilicate fibres with mullite bulk composition. II. Kinetics of mullite formation. Journal of the European Ceramic Society, 1996, 16(2): 287-292.

[17] RICHARDS E A, GOODBRAKE C J, SOWMAN H G. Reactions and microstructure development in mullite fibers. Journal of the American Ceramic Society, 1991, 74(10): 2402-2409.

[18] HULING J C, MESSING G L. Hybrid gels for homoepitactic nucleation of mullite. Journal of the American Ceramic Society, 1989, 72(9): 1725-1729.

[19] HULING J C, MESSING G L. Epitactic nucleation of spinel in aluminosilicate gels and its effect on mullite crystallization. Journal of the American Ceramic Society, 1991, 74(10): 2374-2381. 